\title{
Spatial and Social Networks in Organizational Innovation
}

Jean D. Wineman

Felichism W. Kabo

Gerald F. Davis

University of Michigan

\begin{abstract}
Research on the enabling factors of innovation has focused on either the social component of organizations or on the spatial dimensions involved in the innovation process. But no one has examined the aggregate consequences of the link from spatial layout, to social networks, to innovation. This project enriches our understanding of how innovation works especially in highly innovative organizations by exploring the social dimensions of innovation as they are embedded in a specific spatial milieu. Workspace layout generates spatial boundaries that divide and reunite built space. These boundaries create relations of accessibility and visibility that integrate or segregate behaviors, activities, and people. As built space structures patterns of circulation, copresence, coawareness, and encounter in an organization, these interrelationships become fundamental to the development of social networks, especially those networks critical to the innovation process. This article presents a review of the knowledge bases of social network and spatial layout theories, and reports on a preliminary study of the effects of spatial layout on the formation and maintenance of social network structure and the support of innovation.
\end{abstract}

Keywords: office design; network analysis; space syntax; productivity

\section{Introduction}

Research on the enabling factors of innovation has focused on either the social component of organizations or on the spatial dimensions involved in the innovation process. Burt $(2000,2003,2004)$ has shown how the shape of an individual's network is related to his or her subsequent involvement in

Authors' Note: Please address correspondence to Jean D. Wineman, A. Alfred Taubman College of Architecture and Urban Planning, University of Michigan, 2000 Bonisteel Blvd., Ann Arbor, MI 48109-2069; e-mail: jwineman@umich.edu. 
innovations. Allen $(1977,2000)$ addressed the effect of spatial distance on communication, an important prerequisite for innovation. He documented the famous "Allen curve" in an R\&D facility showing the rapidly declining likelihood of communication between two engineers as the distances of their offices increased. We know of only a couple of studies (Kabo, 2006; Peponis et al., 2007) that have examined the aggregate consequences of the link from spatial layout, to social networks, to organizational outcomes such as innovation, efficiency and efficacy, and productivity. This article presents preliminary work to enrich our understanding of how innovation works in highly innovative organizations by exploring the social dimensions of innovation as they are embedded in a specific spatial milieu.

Workspace layout generates spatial boundaries that divide and reunite built space. These boundaries create relations of accessibility and visibility that integrate or segregate behaviors, activities, and people. As built space structures patterns of circulation, copresence, coawareness, and encounter in an organization, these interrelationships become fundamental to the development of social networks, especially those networks critical to the innovation process. For example, spatial layout influences the informal communication networks critical to creativity within organizations. Allen (2000, p. 153) found that this type of communication is "the most unpredictable and most difficult ... to manage. It is also the most affected by architecture. Since most of this communication occurs during chance encounters, architecture can strongly promote or impede such occurrences."

This article presents a review of the knowledge bases of social network and spatial layout theories, and reports on a preliminary study of the effects of spatial layout on the formation and maintenance of social network structure and the support of innovation.

\section{Relation to the Present State of Knowledge in the Fields}

\section{Innovation}

"Innovation" is a concept studied in many disciplinary contexts, leading to a multiplicity of definitions, including psychological, sociological, and economic ones (Brown \& Ulijn, 2004). The multiplicity of meanings of innovation is partly attributable to the disciplinary biases of innovation researchers.

So, how do we define innovation? The popular consensus is that innovation is associated with novelty-that which is new. This would favor radical change over incremental change. Brown and Ulijn (2004) give a minimum 
definition of innovation as "creating something new and implementing it successfully at a market," and the novel creation could be "processes, products and services and technology" (p. 2; Van de Ven, 1986). Porter and Stern (1999) define innovation as "the transformation of knowledge into new products, processes, and services" (p. 12). These are just two of the many definitions of innovation. Rickard (2003) astutely notes that "we need hardly be surprised that innovation, a word forced to stand for activities across such a rich set of categories, resists a unified yet coherent treatment" (p. 1094).

\section{Social Networks}

Despite all the literature on innovation, key questions on the origin of innovation remain unanswered. One of these key issues is whether innovation is a function of the social networks in which actors are embedded, individual actor agency, or a combination of the two. This issue can be explored through network analysis.

Social network analysis (SNA) may be defined as "the disciplined inquiry into the patterning of relations among social actors, as well as the patterning of relationships among actors at different levels of analysis" (Breiger, 2004). Davis (2006) notes that a simple definition of a network is that it consists of nodes and ties, the relationships among the nodes. And nodes can refer to actors - which can be persons, teams, or organizationsor entities, such as neurons or abstract ideas. Ties "connect pairs of actors and can be directed (i.e., potentially one-directional, as in giving advice to someone) or undirected (as in being physically proximate) and can be dichotomous (present or absent, as in whether two people are friends or not) or valued (measured on a scale, as in strength of friendship). A set of ties of a given type . . constitutes a binary social relation, and each relation defines a different network (e.g., the friendship network is distinct from the advice network ....)" (Borgatti \& Foster, 2003, p. 992).

We will use the concept of social capital to explain how network analysis can be applied in innovation research. Studies exploring social capital typically embody two things: the application of a potent technology, network analysis, to make predictions related to a critical outcome, performance. On the latter, social capital promises "more rigorous and stable models, describing why certain people and organizations perform better than others" (Burt, 2000, p. 2). On the former, social capital has been used to explain how social structure defines advantages in the creation of good ideas. Granovetter (1973) observed that people operate in groups with strong within-group ties, with a high velocity of information flow between 
group members. But the information is redundant, meaning that new ideas must come from the weak ties connecting separate groups. Burt (1992) proposed that weak ties indicate the presence of structural holes, or nonredundant contacts. Advantages accrue to actors who span structural holes, the assumption being that information, beliefs, and behaviors are more homogenous (hence redundant) within than between groups. Naturally, people are more focused on within-group activities, creating holes in between-group information flow. This creates opportunities for people with between-group contacts to broker information flow across structural holes. Brokerage is social capital, as brokers gain a competitive advantage in "creating value with projects that integrate otherwise separate ways of thinking or behaving" (Burt, 2003, p. 3).

Broker networks at their best are "large, sparse, non-hierarchical networks rich in opportunities to broker connections across structural holes. This is the network structure associated ... with more creativity and innovation" (Burt, 2000, p. 68). However, although these networks enhance the generation of novel ideas, they pose an action problem. Burt (2004) and others have found that although structural holes may lead to the generation of novel ideas, there is no evidence of successful action on these ideas. The dispersed, unconnected people around structural holes are more difficult to mobilize around new ideas, making implementation harder. This constitutes the action problem of structural holes.

Whereas structural holes pose an action problem, dense networks have an idea problem: they foster coordination and implementation, but do not engender generation of new ideas. The action problem of structural holes and the idea problem of dense networks cloud the conceptual understanding of the true antecedents of innovation in a range of contexts as innovation is an output of people and ideas. Obstfeld (2005) reconciles this fundamental tension between the two problems by proposing a tertius iungens strategic orientation to account for the joining activity needed for innovation because "innovation involves a joining of people in both sparse and dense networks to produce the coordinated action that leads to innovation" (p. 102). An actor with a tertius iungens orientation seeks to connect disconnected individuals in one's social network, or to enhance coordination between connected individuals. This is in contrast to the tertius gaudens orientation, where the actor benefits by exploiting the information and control benefits accruing from spanning the structural hole between disconnected individuals.

Obstfeld tested the significance of the tertius iungens orientation through a study on an automotive design firm. The findings from his study provided "strong evidence that a tertius iungens orientation, social knowledge, and 
social network density are independent predictors of innovation involvement within the firm" (p. 120). But Obstfeld qualifies these findings, noting that the importance of dense networks in his study may be specific to the automotive design process. Many of the innovations in Obstfeld's study were incremental, or "constituted solutions to well-structured problems. The radical ideas that precede radical innovations may result from the novel information available in sparse [non-dense] networks" (p. 123).

\section{Spatial Layout}

Earlier literature on the effect of spatial location on work and innovation were coarse-grained. Allen's classic curve showed how the likelihood of collaboration/communication dropped significantly for up to 30 meters and then stayed flat, independent of actual distance. Olson et al. (2002) showed that productivity of software developers doubled when they were radically collocated, as opposed to housed in a cubicle farm. Recent years have seen the development of more sophisticated analytic techniques for describing spatial layouts and their properties. One such technique, which we have applied in the research reported in this article, is space syntax.

The techniques for the analysis of spatial form or space syntax analysis were developed by Bill Hillier and his colleagues at University College London (Hillier and Hanson, 1984). Syntax analysis techniques characterize spatial systems on the basis of the ways in which spaces are related to other spaces within a larger system, rather than metric distance. For example, a particular room may be 10 meters from another room, but perhaps more importantly for use patterns, the room may have a direct connection to the other room, or one may have to change direction several times to reach that room. Syntactically, a system of spaces is more integrated if spaces can be easily reached (require fewer changes of direction) from one another, or more segregated if one must make many changes of direction to move from each space to every other space in the system.

Syntax analysis techniques can be applied to building or urban layouts to produce quantitative measures of the attributes of spatial layout. The analysis represents a spatial system as either a system of paths of sight or movement or as a system of visibility polygons or isovists (what can be seen from a particular vantage point). The paths of sight or movement (axial lines) are drawn to cover the entire floor plan and to establish all spatial connections. Isovists are generated to capture the visible spatial area and are drawn from each vantage point on an orthogonal grid [set at a chosen level of resolution] that is superimposed on the floor plan. For each of these 
representations, syntax analysis involves the study of patterns of connections, both in terms of the relationship of each spatial unit (axial line or visibility polygon) to its immediate neighbors measured by connectivity, and by the relationship of each spatial unit or axial line to the entire set of axial lines that constitutes the spatial system being studied, measured by integration. Note that connectivity is also known as "degree" in SNA, or simply how many direct contacts one has (Davis, 2006). Taking axial lines as an example, as a global measure, integration (also known as "closeness" in SNA, in effect, the mean shortest path between this node and all other nodes [Davis]) describes how easily (traversing the fewest number of lines) all other axial lines can be reached from a given line. For our purposes of comparing spatial and social data, in addition to characterizing spatial systems as a whole or subparts, we also need to describe spatial relations between individuals. To obtain dyadic spatial data, step-depth measures the number of turns (or changes in the visibility polygon) that it takes to see from one location to another location on the plan and represents how visually connected or isolated individuals are from one another. Step-depth is known as geodesic (or distance) in SNA, or the length of the shortest path between two actors (Davis).

Research indicates that spatial organization affects the generation and distribution of movement patterns in space, and unplanned encounters between occupants (Hillier \& Penn 1991; Peatross \& Peponis, 1995; Peponis, 1985; Serrato \& Wineman, 1999). In describing the different cultures of two research laboratories, Hillier and Penn found that the properties of the spatial layout of movement corridors (whether near or far from work areas) will affect whether communication tends to spread among research groups, a pattern the authors suggest has greater potential to sustain creative awareness on a more global scale. A study of two institutions for design education (Peatross and Peponis, 1995) develops similar themes, suggesting two ways in which this function of layout may complement formal educational pedagogy: first, through creating opportunities for awareness and communication across the fields of study represented at a school; second, through qualifying the pedagogical process within a field, by fostering a sense of comparison, definition through difference, and even creative competition between tutor-centered groups.

Grajewski (1993) reports strong correlations between interaction (the number of people talking as a proportion of the number of people observed) and integration in six office environments in the United Kingdom, the United States, and Sweden. After taking density into account, it was shown that more integrated office segments (floors or relatively autonomous 
wings) are more interactive. Correlations between integration and interaction were also found within individual office complexes, such as the Scandinavian Airlines System headquarters building in Stockholm. Serrato and Wineman (1999) investigated the relationship between the layout of two research and development facilities and communication patterns among research scientists. Although the two units have different layouts, the strongest predictor of communication for both units was found to be the extent to which scientists were linked to locally integrated corridors and the interface of this local system with the global spatial system. For both labs, the spatial layout supported localization. In one lab, localization reflected patterns of organizational subdivision by knowledge area, maintained apart from global spatial movement. In the other lab, localization mixed knowledge areas, and created collaborative interface across the global spatial system. Penn, Desyllas, and Vaughan (1999) trace the spatial culture of two organizations, an energy utility and an advertising agency, not only in detailed behavioral mapping but also in the nuances of behavior. The aim was to understand the mechanisms whereby the more generic functions of layout, such as the distribution of movement, affect the more critical and less easily observable aspects of function, such as useful communication. The study provides evidence that integration is correlated to the perceived value of conversation, based on a comparison between departments of a single organization and a comparison across a small sample of organizations.

Emergent technologies have contributed to new forms of interaction in offices that are significant influences on the formation of organizational culture. Spiliopoulou and Penn (1999), in a study of a London-based corporation, found that although electronic communications are aspatial, physical space plays an important role in the generation and practice of e-mail and telephone communication. They conclude that e-mail and telephone communication can link workers who are spatially isolated but only under conditions where management tasks require such communication. In two recent studies, Baym, Zhang, and Lin (2004) found that the more college students use e-mail to support social relationships, the more they use telephone and face-to-face communication to support these same social relationships. Kossinets and Watts (2006) are encouraged by these and other studies they cite to use only e-mail communication to infer underlying social networks.

As suggested previously, few studies have examined the interrelationships between social and spatial networks as they relate to performance or productivity outcomes. Kabo (2006) studied patterns in the interorganizational relationships (social networks) and spatial location (based on an analysis of space syntax axial lines) of a sample of 46 key organizations in 
the Kenyan housing sector. The research suggests that the spatial context as it interacts with the social context influences the nature of an organization's involvement in housing provision. This analysis was part of a larger effort to understand the role network position plays in explaining the relative influence and efficiency of housing sector organizations. A particularly relevant study to office organizations is the one recently published by Peponis and his colleagues (2007). Using social and spatial network analysis techniques applied to the study of an organization before and after a move to new premises, this study found that layout can contribute to the density of social interactions and may potentially contribute indirectly to productivity through the support of idea-generating communication.

Clear paradigmatic ideas have emerged from more detailed spatial analyses. First, the correlation between integration and movement patterns is treated as an underlying principle. Second, movement and coawareness by-products of movement are treated as a foundation on which particular patterns of encounter, interaction, or communication may develop. These patterns of spatial and social contact are key factors in research on innovation, specifically the way in which organizational structure inhabits space generatively, sustaining otherwise unplanned patterns of awareness, communication, and interaction.

\section{Preliminary Research Study}

This article reports on a preliminary study of the effects of spatial layout on the formation and maintenance of social network structure and the support of innovation in a professional school at the University of Michigan. The professional school is a good setting for a pilot study of this type because the school has an explicit policy of allocating office space across academic departmental affiliations to promote cross-disciplinary collaboration. Coauthorship is a good indicator of successful collaboration in such a context. Consequently, we treated academic publications as an index of innovation, and coauthorship as an expression of network relations. See networks of selected actors and their office locations in Figure 1.

The purpose was to look at the relationship between innovation, social networks, and spatial layout. But first, we must note that there were some limitations to our pilot study. The first limitation is that although people do not move often, office location is somewhat endogenous, particularly over the 6-year time period-people might move closer to coauthors. The second limitation is that we measured the outcome-coauthorship-but not 


\section{Figure 1}

\section{Ego Networks of Select High-Coauthoring Actors Mapped Onto Physical Space}

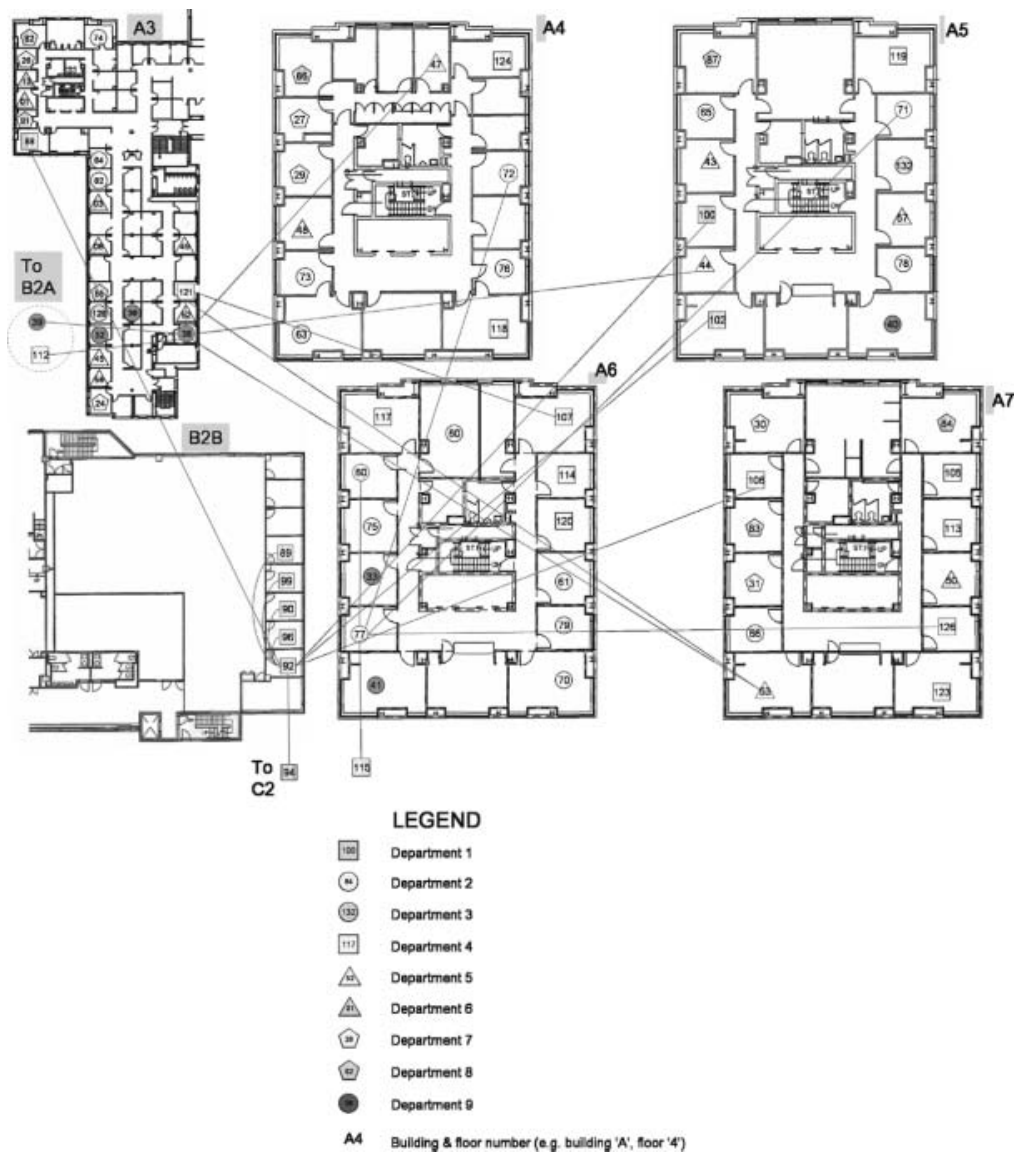

intermediate steps like social/work networks that have not (yet) resulted in publication. Because we did not measure social/work networks independently, we inferred these from patterns of coauthorship. In our pilot, the model was as follows: probability of a pair coauthoring $=f$ (spatial distance; same department), with the unit of analysis being all possible dyads and 
controls for random effects by person and department. Thus, our pilot study explored the effects of space on interaction leading to measurable performance. We were not able to directly address the three-way interaction between space (syntactic measures), social networks (network measures), and organizational performance (coauthored publications).

Our sample consisted of tenured and tenure-track professors who had coauthored with another faculty member from the years 2000-2005, giving a sample size of 82 . Coauthorship was simply determined by analyzing the online curriculum vitae (CVs) of the professional school's professors and determining authorship on the publications listed in the CVs. We created a coauthorship database that was then used to build a coauthorship matrix. This matrix was analyzed using SNA software (InFlow and Ucinet) and a coauthorship network map was generated (Figure 2) in addition to SNA measures, including network density, network structural holes, and centrality. Tie strength was determined by the number of articles coauthored, and the existence of a tie was determined by coauthorship.

The first step in spatial layout analysis is the creation of an axial map. An axial map consists of a set of the fewest and longest paths (axial lines) of sight and movement that cover a layout making all possible connections between individual spaces and rooms. ${ }^{1}$ Using this map, we applied the computer-based analysis program, Depthmap, to calculate a number of global and local spatial measures, including integration, connectivity, and step-depth. The integration analysis is shown in Figure 3 (low values shown in blue and high values shown in red). For the purposes of comparing the spatial data with the social data, it was essential that we obtain spatial data of a dyadic nature. Therefore, we generated step-depth data for all the 82 professors in our sample. More precisely, we calculated the step-depth between the respective offices for each faculty member (because step-depth is a measure of spatial distance).

Finally, we performed regression analyses on our social and spatial data. The model is a logistic regression where the sample is all possible dyads (pairs of faculty); the dependent variable is whether they coauthored one or more papers together during the 5-year period studied. The main effects in the model are samedept and distance (where samedept is "are they in the same department," distance is step-depth (which is 0 if you are on the same hallway), and there are controls for each department but one, with Department 1 being the omitted category). We regressed the likelihood of coauthorship on the distance between coauthors, a dummy variable for being in the same department, and a set of dummy variables for the departments of each of the pair. The model includes clustering for nonindependent 


\section{Figure 2}

\section{Professional School Coauthor Network by Department}
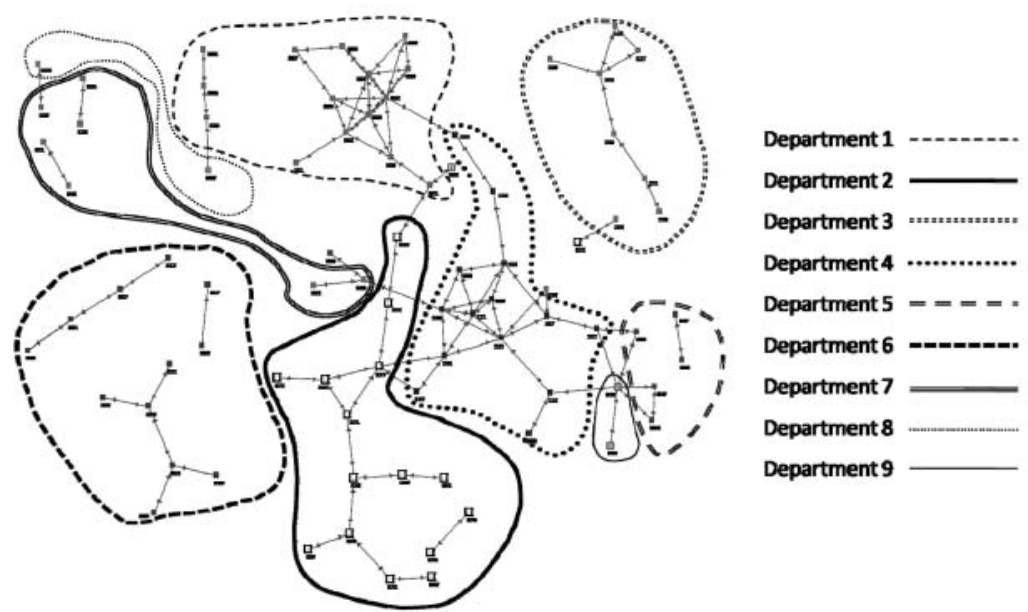

observations (in this case, the first member of the dyad). That is, the error term is corrected for nonindependence of observations. Results indicated that being in the same department is by far the best predictor $(p=.000)$, but that distance had a significant negative effect $(p=.010)$ - that is, the longer the distance, the less the likelihood of coauthorship-after controlling for the departments of the various authors. So, for instance, actors from Department 6 are significantly less likely to coauthor with others in their department than those from Department 1, whereas Department 9 actors are significantly more likely than those from Department 1 to coauthor. Therefore, social (departmental affiliation) and spatial (step-depth) variables do have a significant effect on coauthorship.

We were also interested in determining the extent to which other aspects of spatial layout might affect innovation (measured by total number of articles coauthored). We performed exploratory analyses (linear regressions) on spatial measures and found that there was a significant relationship ( $p=$ .038) between integration and innovation. This suggests that the higher the 


\section{Figure 3}

\section{Integration Axial Map (left), and Professional School Floor Plans (right)}

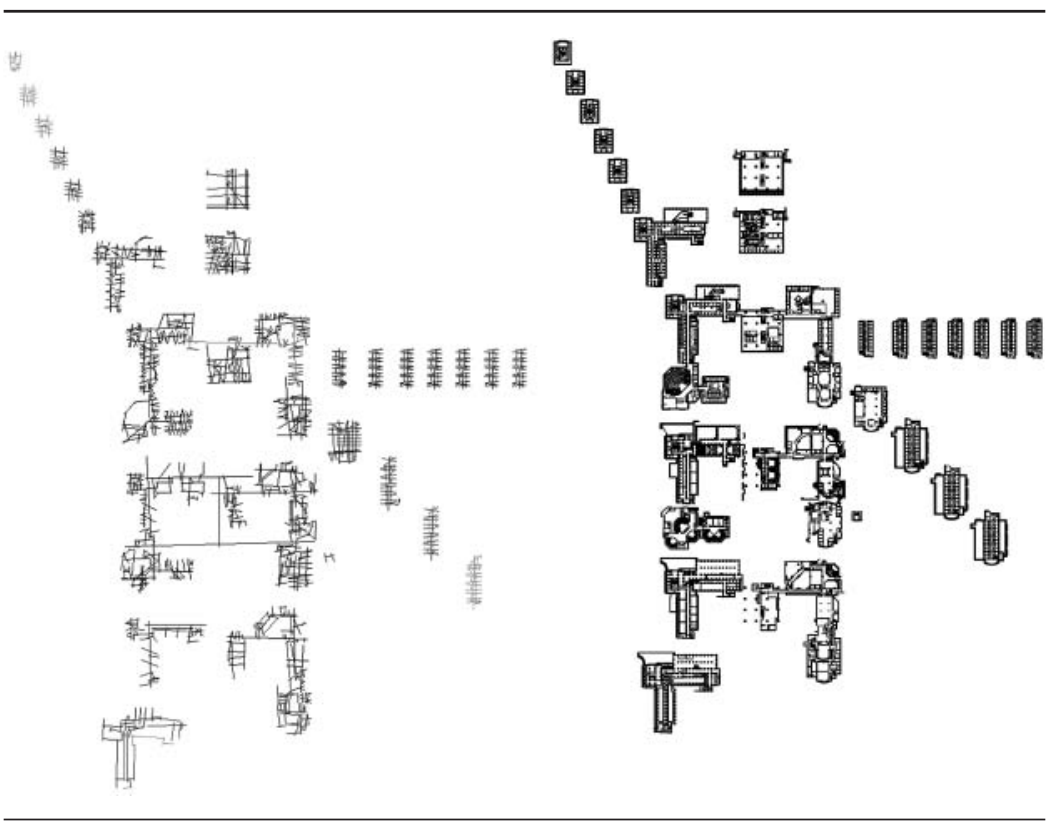

Table 1

Regression of Social and Spatial Variables on Coauthorship

\begin{tabular}{lcccccc}
\hline Coauth 2 & Odds Ratio & Robust $S E$ & $z$ & $p>|z|$ & \multicolumn{2}{c}{$95 \%$ Confidence Interval } \\
\hline samedept & 48.226 & 12.442 & 15.02 & .000 & 29.085 & 79.961 \\
distance & 0.969 & 0.012 & -2.57 & .010 & 0.946 & 0.993 \\
& & & & & & \\
deptII-1 & 0.422 & 0.102 & -3.55 & .000 & 0.262 & 0.679 \\
deptIII-1 & 0.420 & 0.188 & -1.94 & .053 & 0.174 & 1.010 \\
deptIV-1 & 0.966 & 0.254 & -0.13 & .896 & 0.577 & 1.619 \\
deptV-1 & 1.080 & 0.549 & 0.15 & .879 & 0.399 & 2.922 \\
deptVI-1 & 0.336 & 0.098 & -3.73 & .000 & 0.190 & 0.596 \\
deptVII-2 & 0.745 & 0.232 & -0.95 & .343 & 0.405 & 1.370 \\
deptVIII-1 & 1.347 & 1.173 & 0.34 & .732 & 0.245 & 7.420 \\
deptIX-2 & 8.337 & 5.387 & 3.28 & .001 & 2.349 & 29.582 \\
\hline
\end{tabular}

Note: Logistic regression: number of observations $=6162 ;$ Wald $\chi^{2}(10)=312.48 ;$ prob $>\chi^{2}=.0000$; $\log$ pseudo-likelihood $=-539.853$; pseudo $R^{2}=0.325$ ( $S E$ adjusted for 79 clusters in auth 2). 
Table 2

Regression of Integration on Number of Articles Coauthored

\begin{tabular}{|c|c|c|c|c|}
\hline Source & SS & & & MS \\
\hline Model & 168.584 & & & 168.584 \\
\hline Residual & 2905.492 & & & 37.734 \\
\hline Total & 3074.076 & & & 39.411 \\
\hline Articles & Coeff. & $S E$ & $t$ & $P>|t|$ \\
\hline Integration & 6.813 & 3.223 & 2.11 & .038 \\
\hline Constant & 1.769 & 1.832 & 0.97 & .337 \\
\hline
\end{tabular}

Note: SS = Sum of Squares; MS = Mean Squares; Number of observations = 79 (The sample size was revised downward from 82 to 79 to account for the three professors whose offices are in other academic units within the university.); $F(1,77)=4.47$; prob $>F=.038 ; R^{2}=.055$; adjusted $R^{2}=.044 ;$ RMSE $=6.143$.

extent to which a faculty member's office is located along a corridor that is well connected (integrated) to all the other corridors in the department, the greater the likelihood of coauthorship within the department.

\section{Summary and Conclusions}

Although a purely exploratory exercise, the findings of the professional school study are nonetheless informative. The professional school has an explicit policy of dispersing people across academic departments to promote cross-disciplinary collaboration. Our findings suggest that this was an effective organizational strategy. Although departmental allegiances were most influential in coauthorship, nevertheless, location was significant. The farther (as measured by step distance) apart faculty offices were located, the less likely the faculty members were to coauthor. Aspects of social networks and spatial layout combine to provide a good indicator of successful collaboration.

Furthermore, the research indicates that controlling for departmental affiliation, faculty whose offices are located along well-connected corridors have a greater likelihood of coauthorship. This finding suggests that office layouts that are more syntactically integrated (well connected) will promote communication, leading to productive outputs across disciplinary boundaries (similar to the findings of Peponis et al., 2007). Spatial layout apparently plays a strong supportive role in the formation and maintenance of social relations that ultimately are linked to innovation. 
The research explores potential opportunities to guide the social and organizational structure and spatial layout of workers to create more effective, efficient, and innovative work processes. In the example of the professional school, innovations as represented by cross-disciplinary joint-authored publications would likely be considered incremental innovation rather than radical innovation. As previously cited, in his study of an automobile design firm that follows this same model, Obstfeld concludes that connecting disconnected individuals within social networks and enhancing coordination between connected individuals are strong predictors of innovation. For the professional school, even this exploratory study suggests that there are potent spatial factors at work that enhance these collaborations: the school's approach of dispersing faculty across academic departments, and spatial layouts that act to densify across individuals or groups.

This area of exploration has broader impacts to organizational administrators/ managers, workplace designers, and architects in producing effective work environments. Workplace spatial layout is often considered a management tool to promote the aims of the organization, and can be a powerful tool for shaping organizational culture and achievement.

\section{Note}

1. In multistory buildings such as those at the professional school, the different floor levels are connected by circulation spaces like stair areas and elevators.

\section{References}

Allen, T. J. (1977). Managing the flow of technology: Technology transfer and the dissemination of technological information within the $R \& D$ organization. Cambridge, MA: MIT Press.

Allen, T. J. (2000). Architecture and communication among product development engineers. Proceedings of the 2000 IEEE (pp. 153-158). Engineering Management Society, 2000.

Baym, N. K., Zhang, Y. B., \& Lin, M.-C. (2004). Social interactions across media. New Media \& Society, 6, 299-318.

Borgatti, S. P., \& Foster, P. C. (2003). The network paradigm in organizational research: A review and typology. Journal of Management, 29, 991-1013.

Breiger, R. L. (2004). The analysis of social networks. In M. Hardy \& A. Bryman (Eds.), Handbook of data analysis. London: Sage.

Brown, T. E., \& Ulijn, J. M. (2004). Innovation, entrepreneurship and culture: The interaction between technology, progress and economic growth. Northampton, MA: Edward Elgar.

Burt, R. S. (1992). Structural holes: The social structure of competition. Cambridge, MA: Harvard University Press. 
Burt, R. S. (2000). The network structure of social capital. In R. I. Sutton \& B. M. Staw (Eds.), Research in organizational behavior. Greenwich, CT: JAI Press.

Burt, R. S. (2003). Social origins of good ideas. Retrieved January 13, 2006, from http://web.mit.edu/sorensen/www/SOGI.pdf

Burt, R. S. (2004). Structural holes and good ideas. American Journal of Sociology, 110, 349-399.

Davis, G. F. (2006). Networks in and around organizations. In W. R. Scott \& G. F. Davis (Eds.), Organizations and organizing: Rational, natural, and open systems perspectives. Upper Saddle River, NJ: Prentice Hall.

Grajewski, T. (1993). The SAS head office-Spatial configuration and interaction patterns. Nordic Journal of Architectural Research, 2, 63-74.

Granovetter, M. S. (1973). The strength of weak ties. American Journal of Sociology, 78, 1360-1380.

Hillier, B., \& Hanson, J. (1984). The social logic of space. Cambridge: Cambridge University Press.

Hillier, B., \& Penn, A. (1991). Visible colleges: Structure and randomness in the place of discovery. Science in Context, 4(1), 23-49.

Kabo, F. W. (2006). Organizational analysis, part III: Spatial and socio-spatial relationships among housing sector organizations. In Low-cost housing design and provision: A case study of Kenya (Chap. 10). PhD dissertation, University of Michigan.

Kossinets, G., \& Watts, D. (2006). Empirical analysis of an evolving social network. Science, 311, 88-90.

Obstfeld, D. (2005). Social networks, the Tertius Iungens orientation, and involvement in innovation. Administrative Science Quarterly, 50, 100-130.

Olson, J .S., Teasley, S., Covi, L., \& Olson, G. M. (2002). The (currently) unique value of collocated work. In S. Kiesler \& P. Hinds (Eds.), Geographically distributed work. Cambridge, MA: Academic Press.

Peatross, D., \& Peponis, J. (1995). Space, education and socialization. Journal of Architectural and Planning Research, 12, 366-385.

Penn, A., Desyllas, J., \& Vaughan, L. (1999). The space of innovation: Interaction and communication in the work environment. Environment and Planning B: Planning and Design, 26, 193-218.

Peponis, J. (1985). The spatial culture of factories. Human Relations, 38(April), 357-390.

Peponis, J., Bafna, S., Bajaj, R., Bromberg, J., Congdon, C., Rashid, M., et al. (2007). Designing space to support knowledge work. Environment and Behavior, 39(6), 815-840.

Porter, M. E., \& Stern, S. (1999). The new challenge to America's prosperity: Findings from the innovation index. Washington, DC: Council on Competitiveness.

Rickard, T. (2003). The future of innovation research. In L. V. Shavinina (Ed.), The international handbook on innovation. Oxford: Elsevier Science.

Serrato, M., \& Wineman, J. (1999). Spatial and communication patterns in research \& development facilities. Second International Space Syntax Symposium, 1, 11.1-11.8.

Spiliopoulou, G., \& Penn, A. (1999). Organizations as multi-layered networks: Face to face, e-mail and telephone interaction in the workplace. Second International Space Syntax Symposium, 1,13.1-13.24.

Van de Ven, A. H. (1986). Central problems in the management of innovation. Management Science, 32, 590-607. 
Jean D. Wineman (DArch [1977] and MUP [1973], University of Michigan) is Professor of Architecture at the Taubman College of Architecture and Urban Planning, University of Michigan. Her research interests focus on the visual and spatial properties of buildings and urban spaces; social, cultural, and behavioral studies; and environmental cognition.

Felichism W. Kabo (PhD [2006], MSc [2004], and MArch [2002], University of Michigan) is a postdoctoral scholar at the Taubman College of Architecture and Urban Planning, University of Michigan. His research focuses on spatial properties of buildings and urban spaces; user perceptions of buildings and materials; design, sociocultural, and organizational elements in low-cost housing provision; and social networks.

Gerald F. Davis (PhD [1990] and MA [1987], Stanford University) is Wilbur K. Pierpont Collegiate Professor of Management at the Ross School of Business, University of Michigan. His research focuses on social networks, organization theory, corporate governance, and the social effects of financial market expansion. 